\title{
THERMAL EXPANSION OF A FEW STEELS
}

\author{
By Wilmer Souder and Peter Hidnert
}

ABSTRACT

Thermal expansion data are given on 28 specimens of iron and steel. Most of the specimens were heated above $900^{\circ} \mathrm{C}$. Tables and curves are used to summarize the results. The tests were made by direct measurements of the length changes and not relative to some other substance or element. The observations were made without disturbing the furnace or specimen and therefore give correct relative values for all temperature intervals.

Variations in length changes, contraction and expansion reversals while passing through the critical regions, and similar changes are compared and discussed.

An attempt is made to throw some light on the magnitude of the tendency toward warping or surface cracking as related to the rate of cooling and width of critical regions.

Expansion coefficients are tabulated for the various alloys and for various temperature intervals.

Data on one specimen of vacuum electrolytic iron and one of gray cast iron are recorded. The length changes incident to the drawing of a sample of hardened steel are shown in a curve.

A brief review of some of the previous work on expansion is included.

\section{CONTENTS}

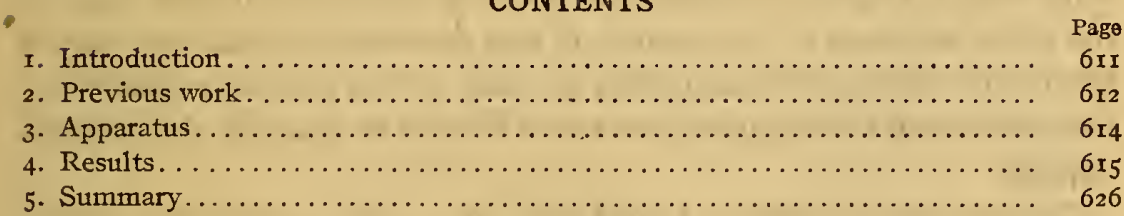

\section{INTRODUCTION}

The anomalous expansion of iron and certain steels when heated over the range 600 to $1000^{\circ} \mathrm{C}$ has been known for a number of years. The structural changes taking place at or near these points of nonuniform expansion have been given considerable attention within the last decade. In addition to the irregularities in expansion, which are influenced by these changes, there are numerous other physical properties which are modified during the passage through the above temperature range. Among the effects which have been used to more accurately chart this region are: Changes in electrical, magnetic, elastic, and thermal 
properties, changes in microstructure, hardness, and rate of expansion.

The present paper presents additional data on the last-named property, and while the emphasis will not be placed on the structural or molecular phases of ten accompanying the variations in expansivity, it is felt that the data may be of sufficient accuracy to warrant their use for certain parts of such study should one care to make such use of them. The results represent direct measurement of expansion on a number of selected specimens and are perhaps above the average in accuracy for direct, continuous measurements of thermal expansion.

The principles of hardening and heat treatments of steel are dependent to some degree upon the proper handling while within the above range. Our comments on the dimensional changes will bear upon the physical effects which may be expected to accompany these dimensional irregularities. The shrinkage, warping, surface cracking, and usual lack of highest accuracy of dimensions met in heat-treated work render data on expansion and contraction worthy of careful consideration.

\section{PREVIOUS WORK}

Svedelius, Le Chatelier, Charpy and Grenet, Guillaume, Driesen, Honda, and other investigators have done work on the thermal expansion of iron and steel. Since a satisfactory review of their work would require considerable space in comparison with that of the other sections of this paper, it was decided to point out only a few of the results or conclusions of some of the previous observers. For additional information, reference should be made to the original papers.

CHARPY AND GRENET ${ }^{1}$ found that, if small samples of medium steels (o.6 to I per cent C) and of high-carbon steels were heated above $900^{\circ} \mathrm{C}$ and quenched in water, the expansion curves of the former show abrupt contraction of about I per cent between 250 and $350^{\circ} \mathrm{C}$, and the curves of the high-carbon steels indicate two contractions, one at about 150 and the other at $300^{\circ} \mathrm{C}$.

In a later paper ${ }^{2}$ these authors compare results obtained in the critical regions of steels by three different methods. The following tables show the comparative values:

\footnotetext{
${ }^{1}$ Charpy and Grenet, C. R., 136, pp. 92-94; Jan. x2, x903.
}

${ }^{2}$ Charpy and Grenet, C. R., 139, pp. 567-568; Oct. 10, 1904. 
TABLE 1.-Comparison of Electrical Resistance and Dilatation Methods (Charpy and Grenet)

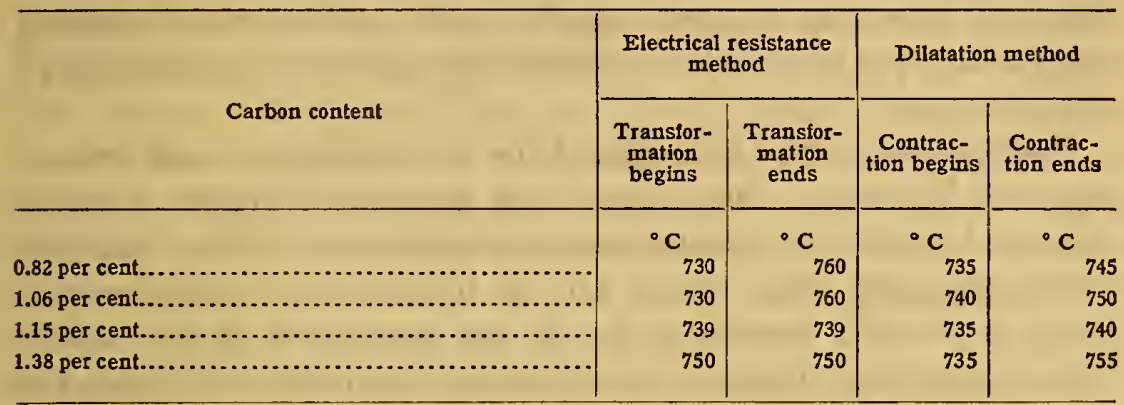

TABLE 2,-Comparison of Thermoelectric and Dilatation Methods (Charpy and Grenet)

\begin{tabular}{|c|c|c|c|c|}
\hline \multirow[b]{2}{*}{ Carbon content } & \multicolumn{2}{|c|}{ Thermoelectric method } & \multicolumn{2}{|c|}{ Dilatation method } \\
\hline & of $\frac{\mathrm{d} E}{\mathrm{~d} t}$ & $\begin{array}{l}\text { Minimum } \\
\text { of } \frac{\mathrm{d} E}{\mathrm{~d} t}\end{array}$ & $\begin{array}{c}\text { Contrac- } \\
\text { tion begins }\end{array}$ & $\begin{array}{l}\text { Contrac- } \\
\text { tion ends }\end{array}$ \\
\hline 0.28 per cent........................... & ${ }^{\circ} \mathrm{C}_{720}$ & ${ }^{\circ} \mathrm{C}_{840}$ & ${ }^{\circ} \mathrm{C}_{720}$ & ${ }^{\circ} \mathrm{C}_{820}$ \\
\hline 0.62 per cent......................... & 700 & 760 & 743 & 760 \\
\hline 0.92 per cent......................... & 700 & 800 & 737 & 760 \\
\hline 1.14 per cent............................ & 708 & 780 & 747 & 760 \\
\hline 1.30 per cent........................................ & 680 & 740 & 725 & 740 \\
\hline
\end{tabular}

Charpy AND CORNU ${ }^{3}$ found that more than I.3 per cent Si in low-carbon steels (o.I per cent $\mathrm{C}, 0.3 \mathrm{Mn}$ ), caused the expansion curve to be almost rectilinear from o to $900^{\circ} \mathrm{C}$, without an indication of a critical region. However, for an alloy containing 0.35 per cent $\mathrm{C}$ and 0.8 per cent $\mathrm{Mn}$, it required 4.5 per cent $\mathrm{Si}$ to show a similar phenomenon.

DRIESEN ${ }^{4}$ states that above the critical region, the coefficients of expansion of carbon steels are practically constant for samples containing less than 0.85 per cent $\mathrm{C}$. This conclusion is in agreement with the results of Charpy and Grenet. ${ }^{5}$ The carbon steel containing 0.33 per cent $\mathrm{C}$ showed the maximum change of length during the critical region. Honda ${ }^{6}$ gives data which are in close agreement with this result, for he found that a steel containing 0.3 I per cent $C$ indicated the maximum contraction in the trans-

\footnotetext{
Charpy and Cornu, C. R., 156, pp. 1240-1243; Apr. 21, 1913.

- Driesen, Ferrum, 11, pp. 129-138, Feb., and pp. 161-169, Mar., 1914; Rev. de Mét., 14, Dp. 683-706, Sept.-Oct., $x 9 \times 7$

${ }^{\circ}$ Charpy and Grenet, C. R., 134, p. 540: 1902.

- Honda, Tơhoku Univ., Sci. Reports, 6, pp. 203-212, Nov., I9I7.
} 
formation region. Driesen also found that steels containing more than 0.65 per cent carbon and quenched from above $900^{\circ} \mathrm{C}$ contract on reheating to about $300^{\circ} \mathrm{C}$, and with steels containing more than I per cent carbon a similar phenomenon is also observed at 100 to $150^{\circ} \mathrm{C}$.

ŌKÔCHI AND SATÔ ${ }^{7}$ determined the growth of gray cast iron on repeated heatings. They found that permanent growth is never produced below the transformation temperature. They state the rapid expansion from about $650^{\circ} \mathrm{C}$ to near the transformation point in the first heating is due to the separation of free carbon from cementite. There are two periods of growth: (I) During the transformation, and (2) at temperatures above the transformation.

Benedicks and others have used certain modifications of the thermal expansive relations to more accurately define transformations in structure, etc. However, since these tests are, in general, only differential tests of expansivity, no claims being made for the absolute values of thermal expansion, or since their application has been limited to metallurgical considerations, it is not within the province of this review to discuss their results.

In many instances we have been prevented from making more detailed comparisons of this work with that of other investigators because of the more complex composition of these steels. While the agreement on carbon content, for example, may be good, the included manganese, vanadium, or chromium may have introduced an unknown effect. These steels were selected with the idea of securing representative specimens rather than any special series of some one alloying element.

\section{APPARATUS}

The apparatus ${ }^{8}$ of the Bureau's expansivity laboratory was used. The specimens were $30 \mathrm{~cm}$ in length and about $\mathrm{I} \mathrm{cm}$ in diameter. The platinum-osmium position wires were placed in. sharp V-notches cut near each end and at right angles to the axis of the specimens. In this manner the oxide which always formed did not influence the longitudinal separation of the vertical position wires. Attempts to gold plate the specimens or to use neutral gases in the furnace were not successful, due perhaps to the occluded gases which could not be displaced; hence the above method of setting the wires in notches was adopted and proved quite satisfactory.

${ }_{7}$ Ōkôchi and Satô, Tokyo Univ. Coll. of Engg., J. ro, pp. 53-71, Feb., 1920.

${ }^{8}$ Described in B. S. Sci. Papers, No. 352. 


\section{RESULTS}

This work (with one or two exceptions) deals with annealed alloys. The specimens were carried through the transformations at a slow rate of temperature change, usually less than I degree per minute. The length changes showed little, if any, lag for temperature variations. Intentional reversals in temperature gradient were accompanied by corresponding reversals in length changes and without a lag of more than 2 minutes. A further confirmation of the uniformity of temperature throughout the entire specimen is found in the sharp breaks in the expansion curves, indicating that practically all parts began the transformation at the same instant. This uniformity of temperature is more readily maintained at high temperatures than at lower temperatures because of the rapid increase in rate of transfer of radiant energy with increase of temperature.

In some instances the tests were disturbed before all data were taken, and in repeating the test only a few points were taken within the range of the previous test. These points usually agreed with the previous test, and in such cases the second run is shown in the curve as a dotted or broken line. (See Fig. 15.) Heating values are indicated by open center circles and cooling values by dark center circles. Where the observations were so numerous that these circles interfered with each other the curve has been omitted. The specimen was watched constantly to see that no smaller variations were overlooked. When the expansion or contraction appeared to be regular these intervening observations were not recorded, or if recorded were not used in the plots.

The cast-iron specimen $\mathrm{S}_{4} 83$ (Fig. ${ }^{17}$ ) is included to show the rapid growth at high temperature. Additional work is being planned to cover this field-more thoroughly. The specimen of hardened steel (Fig. I6) gives a graphical picture of the shrinkages accompanying the drawing process.

Little if any of the above work can be considered as new information. It is perhaps a further confirmation of the findings of other observers, many of which were using entirely different lines of attack. The results will indicate the accuracy and ease with which it is possible to investigate materials at higher temperatures by this method of thermal analysis. Doubtless much of the value of these data will be worked out by those more closely connected with the industrial applications of such.

The results of the expansion measurements are given by the curves of Figs. 2 to 22 and the data relating thereto are assembled 
TABLE 3.-Results of Analyses and Measurements of the Samples PART 1.-RESULTS OF CHEMICAL ANALYSES

\begin{tabular}{|c|c|c|c|c|c|c|c|c|c|}
\hline Lab. No. & C & $\mathrm{Mrn}$ & $\mathbf{P}$ & $\mathbf{S}$ & Si & $\mathrm{Cr}$ & v & N1 & $\begin{array}{l}\text { Miscel- } \\
\text { laneous }\end{array}$ \\
\hline 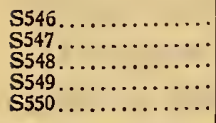 & $\begin{array}{r}0.35 \\
.49 \\
.41 \\
.44 \\
.59\end{array}$ & $\begin{array}{l}1.42 \\
1.21 \\
.64 \\
.57 \\
.92\end{array}$ & $\begin{array}{l}0.013 \\
.05 \\
.052 \\
.013 \\
.024\end{array}$ & $\begin{array}{l}0.057 \\
.050 \\
.061 \\
.033 \\
.033\end{array}$ & $\begin{array}{l}0.20 \\
.12 \\
.086 \\
.161 \\
.25\end{array}$ & $\begin{array}{r}1.00 \\
\cdots \cdots \cdots \\
\cdots \cdots \cdots \\
\cdots \cdots \cdots \\
\cdots \cdots \\
\cdots \cdots\end{array}$ & $\begin{array}{r}0.11 \\
\cdots \cdots \\
\cdots \\
.174 \\
\cdots\end{array}$ & & \\
\hline 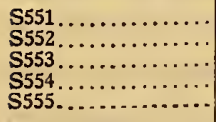 & $\begin{array}{l}.35 \\
.36 \\
.168 \\
.410 \\
.144\end{array}$ & $\begin{array}{r}.08 \\
.46 \\
.01 \\
1.11 \\
.10\end{array}$ & $\begin{array}{l}.010 \\
.011 \\
.010 \\
.053 \\
.03\end{array}$ & $\begin{array}{l}.027 \\
.029 \\
.026 \\
.049 \\
.035\end{array}$ & $\begin{array}{l}.110 \\
.09 \\
.135 \\
.115 \\
.034\end{array}$ & $\begin{array}{l}1.17 \\
2.57 \\
1.50 \\
1.15\end{array}$ & $\begin{array}{r}.14 \\
.12 \\
.39 \\
. .21 \\
.21\end{array}$ & $\begin{array}{r}\cdots \\
3.94 \\
2.00 \\
\ldots \ldots\end{array}$ & Cu 1.85 \\
\hline 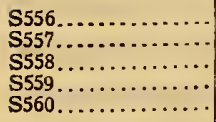 & $\begin{array}{l}.252 \\
.168 \\
.122 \\
.326 \\
.342\end{array}$ & $\begin{array}{l}.06 \\
.08 \\
.05 \\
.78 \\
.28\end{array}$ & $\begin{array}{l}.012 \\
.010 \\
.020 \\
.014 \\
.01\end{array}$ & $\begin{array}{l}.035 \\
.029 \\
.040 \\
.035 \\
.043\end{array}$ & $\begin{array}{l}.007 \\
.038 \\
.846 \\
.094 \\
.094\end{array}$ & $\begin{array}{r}.92 \\
.85 \\
\cdots .82 \\
.89\end{array}$ & $\begin{array}{r}.24 \\
.23 \\
.26\end{array}$ & $\begin{array}{r}\ldots .39 \\
\cdots \\
\cdots\end{array}$ & $\begin{array}{l}\text { Mo. } 64 \\
\text { Cu } 2.70\end{array}$ \\
\hline 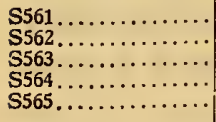 & $\begin{array}{r}.396 \\
.380 \\
.388 \\
.512 \\
30-.40\end{array}$ & $\begin{array}{r}.25 \\
1.17 \\
1.21 \\
.42 \\
. . .\end{array}$ & $\begin{array}{l}.012 \\
.055 \\
.010 \\
.016 \\
. \ldots .\end{array}$ & $\begin{array}{l}.023 \\
.067 \\
.043 \\
.021\end{array}$ & $\begin{array}{c}.095 \\
.10 \\
1.04 \\
1.45 \\
\ldots \ldots \ldots\end{array}$ & 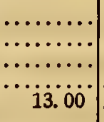 & $\begin{array}{l}\cdots \\
\cdots \cdots \\
\cdots \cdots\end{array}$ & $\mid \begin{array}{r}\ldots \ldots .81 \\
3.67 \\
\ldots \ldots \\
\cdots \cdots\end{array}$ & W 3.96 \\
\hline 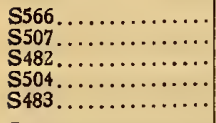 & $\begin{array}{c}.418 \\
.02 \\
1.28 \\
.20 \\
3.08\end{array}$ & $\begin{array}{r}.68 \\
\text { nil } \\
.37 \\
1.10 \\
\ldots \ldots\end{array}$ & $\begin{array}{r}.012 \\
\text { nil } \\
.05\end{array}$ & $\begin{array}{c}.025 \\
.007 \\
.05 \\
.05 \\
. . \cdots\end{array}$ & $\begin{aligned} & .23 \\
& .006 \\
& \cdots \cdots \cdots \\
& \cdots \\
& 1.68\end{aligned}$ & $\begin{array}{c}(\mathrm{Co}, \mathrm{C} \\
.19 \\
.5\end{array}$ & $\begin{array}{l}\text { îi, töta } \\
\cdots \cdots \cdots \\
\cdots \cdots \cdots \\
\cdots \cdots\end{array}$ & $\begin{array}{c}1 \ldots \ldots 0 . \\
\ldots \ldots \ldots \\
\ldots \ldots \ldots\end{array}$ & r \\
\hline 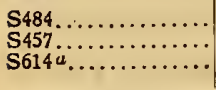 & $\begin{array}{l}.14 \\
.09 \\
.85\end{array}$ & $\begin{array}{l}\text { \%ig } \\
1.00\end{array}$ & $<.02$ & (n........ & $\begin{array}{r}3.70 \\
.25\end{array}$ & $\begin{array}{r}\because 70 \\
.50\end{array}$ & $005-$ & $\begin{array}{l}34.52 \\
.05- \\
\ldots\end{array}$ & W. 45 \\
\hline
\end{tabular}
PART 2.-AVERAGE COEFFICIENTS OF EXPANSION ON HEATING (TEMPER̃ATURES
INDICATED) $\times 10^{\circ}$

\begin{tabular}{|c|c|c|c|c|c|c|c|c|c|c|}
\hline Lab. No. & $\begin{array}{r}25 \text { to } \\
100^{\circ} \mathrm{C}\end{array}$ & $\begin{array}{l}100 \text { to } \\
200^{\circ} \mathrm{C}\end{array}$ & $\begin{array}{r}200 \text { to } \\
300^{\circ} \mathrm{C}\end{array}$ & $\begin{array}{l}300 \text { to } \\
400^{\circ} \mathrm{C}\end{array}$ & $\begin{array}{l}400 \text { to } \\
500^{\circ} \mathrm{C}\end{array}$ & $\begin{array}{r}500 \text { to } \\
600^{\circ} \mathrm{C}\end{array}$ & $\begin{array}{r}600 \text { to } \\
700^{\circ} \mathrm{C}\end{array}$ & $\begin{array}{r}25 \text { to } \\
300^{\circ} \mathrm{C}\end{array}$ & $\begin{array}{r}300 \text { to } \\
600^{\circ} \mathrm{C}\end{array}$ & $\begin{array}{r}25 \text { to } \\
600^{\circ} \mathrm{C}\end{array}$ \\
\hline 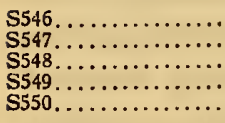 & $\begin{array}{l}12.4 \\
11.3 \\
11.1 \\
11.2 \\
11.1\end{array}$ & $\begin{array}{l}12.8 \\
12.2 \\
12.2 \\
12.4 \\
12.5\end{array}$ & $\begin{array}{l}14.4 \\
14.2 \\
14.3 \\
14.3 \\
14.6\end{array}$ & $\begin{array}{l}15.1 \\
16.3 \\
15.8 \\
15.4 \\
15.4\end{array}$ & $\begin{array}{l}15.9 \\
17.7 \\
15.7 \\
16.4 \\
16.1\end{array}$ & $\begin{array}{l}15.9 \\
15.4 \\
16.0 \\
16.5 \\
16.8\end{array}$ & $\begin{array}{l}16.2 \\
16.7 \\
16.6 \\
16.8 \\
16.6\end{array}$ & $\begin{array}{l}13.3 \\
12.7 \\
12.7 \\
12.7 \\
12.9\end{array}$ & $\begin{array}{l}15.6 \\
16.5 \\
15.8 \\
16.1 \\
16.1\end{array}$ & $\begin{array}{l}14.5 \\
14.7 \\
14.3 \\
14.5 \\
14.6\end{array}$ \\
\hline 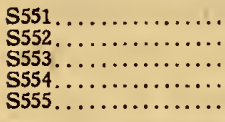 & $\begin{array}{l}11.0 \\
11.8 \\
10.8 \\
11.6 \\
11.2\end{array}$ & $\begin{array}{l}12.3 \\
12.6 \\
11.7 \\
11.9 \\
12.6\end{array}$ & $\begin{array}{l}14.8 \\
14.4 \\
13.5 \\
14.0 \\
13.8\end{array}$ & $\begin{array}{l}15.6 \\
15.1 \\
14.0 \\
16.2 \\
15.6\end{array}$ & $\begin{array}{l}15.9 \\
16.0 \\
14.5 \\
15.7 \\
15.6\end{array}$ & $\begin{array}{l}16.5 \\
16.6 \\
14.4 \\
16.5 \\
16.0\end{array}$ & $\begin{array}{l}16.2 \\
16.8 \\
13.4 \\
16.7\end{array}$ & $\begin{array}{l}12.9 \\
13.1 \\
12.1 \\
12.6 \\
12.7\end{array}$ & $\begin{array}{l}16.0 \\
15.9 \\
14.3 \\
16.1 \\
15.7\end{array}$ & $\begin{array}{l}14.5 \\
14.5 \\
13.3 \\
14.4 \\
14.3\end{array}$ \\
\hline 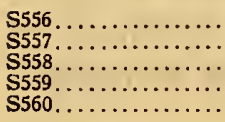 & $\begin{array}{l}11.1 \\
11.3 \\
11.6 \\
10.9 \\
11.6\end{array}$ & $\begin{array}{l}12.0 \\
11.8 \\
12.5 \\
11.5 \\
12.6\end{array}$ & $\begin{array}{l}14.2 \\
13.9 \\
13.7 \\
13.6 \\
14.2\end{array}$ & $\begin{array}{l}15.5 \\
15.3 \\
14.6 \\
15.2 \\
16.0\end{array}$ & $\begin{array}{l}15.9 \\
15.7 \\
15.2 \\
15.1 \\
15.9\end{array}$ & $\begin{array}{l}16.6 \\
16.6 \\
10.0 \\
15.7 \\
16.4\end{array}$ & $\begin{array}{l}16.9 \\
16.1 \\
15.8 \\
16.9\end{array}$ & $\begin{array}{l}12.5 \\
12.5 \\
12.7 \\
12.1 \\
12.9\end{array}$ & $\begin{array}{l}16.0 \\
15.9 \\
15.2 \\
15.3 \\
16.1\end{array}$ & $\begin{array}{l}14.3 \\
14.2 \\
14.0 \\
13.8 \\
14.6\end{array}$ \\
\hline 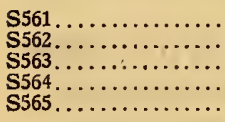 & $\begin{array}{l}11.1 \\
11.2 \\
11.6 \\
10.4 \\
10.0\end{array}$ & $\begin{array}{l}12.0 \\
12.7 \\
12.0 \\
12.1 \\
10.6\end{array}$ & $\begin{array}{l}14.0 \\
14.3 \\
13.2 \\
13.7 \\
12.0\end{array}$ & $\begin{array}{l}15.1 \\
15.2 \\
14.2 \\
15.9 \\
12.6\end{array}$ & $\begin{array}{l}15.7 \\
16.2 \\
15.2 \\
15.7 \\
13.5\end{array}$ & $\begin{array}{l}16.4 \\
16.7 \\
15.6 \\
16.1 \\
13.9\end{array}$ & $\begin{array}{r}16.5 \\
16.4 \\
\cdots \cdots \cdots \\
\cdots 13.7\end{array}$ & $\begin{array}{l}12.5 \\
12.9 \\
12.3 \\
12.2 \\
11.0\end{array}$ & $\begin{array}{l}15.7 \\
16.1 \\
15.0 \\
15.9 \\
13.3\end{array}$ & $\begin{array}{l}14.2 \\
14.5 \\
13.7 \\
14.2 \\
12.2\end{array}$ \\
\hline 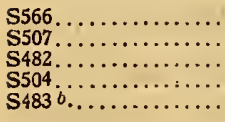 & $\begin{array}{r}9.4 \\
12.0 \\
11.0 \\
12.3 \\
8.4\end{array}$ & $\begin{array}{l}12.0 \\
13.0 \\
11.6 \\
12.9 \\
11.7\end{array}$ & $\begin{array}{l}14.3 \\
14.5 \\
13.6 \\
14.2 \\
14.2\end{array}$ & $\begin{array}{l}15.3 \\
15.3 \\
15.1 \\
15.9 \\
15.6\end{array}$ & $\begin{array}{l}16.4 \\
15.9 \\
16.3 \\
16.2 \\
14.3\end{array}$ & $\begin{array}{c}17.1 \\
16.8 \\
16.1 \\
16.5 \\
\text { Groy }\end{array}$ & $\begin{array}{l}16.8 \\
17.4 \\
17.0 \\
16.8 \\
\text { wing }\end{array}$ & $\begin{array}{l}12.1 \\
13.3 \\
12.0 \\
13.2 \\
11.6\end{array}$ & $\begin{array}{c}16.2 \\
15.9 \\
15.9 \\
16.5 \\
\text { Grow }\end{array}$ & $\begin{array}{r}14.3 \\
14.7 \\
14.1 \\
14.9 \\
\text { ing }\end{array}$ \\
\hline $\begin{array}{l}\mathbf{S} 484 \ldots \ldots \ldots \ldots \ldots \ldots \ldots \ldots \\
\text { S457.............. }\end{array}$ & $\begin{array}{r}3.7 \\
11.1\end{array}$ & $\begin{array}{r}8.4 \\
12.4\end{array}$ & $\begin{array}{l}14.1 \\
13.3\end{array}$ & $\begin{array}{l}16.6 \\
14.0\end{array}$ & $\begin{array}{l}18.4 \\
15.6\end{array}$ & $\begin{array}{l}18.8 \\
16.0\end{array}$ & $\begin{array}{l}19.1 \\
16.9\end{array}$ & $\begin{array}{r}9.2 \\
12.6\end{array}$ & $\begin{array}{l}18.2 \\
15.3\end{array}$ & $\begin{array}{l}13.6 \\
14.0\end{array}$ \\
\hline
\end{tabular}

a Hardened from $80^{\circ} \mathrm{C}$; scleroscope 60 ; shortened I. $2 \mathrm{~mm}$ per meter.

b Expansion values must be considered as approximate only, since the values doubtless contain a percentage of growth in addition to expansion. 
TABLE 3-Continued

PART 3.-CRITICAL REGION DATA

\begin{tabular}{|c|c|c|c|c|c|c|c|c|}
\hline \multirow[b]{2}{*}{ Lab. No. } & \multicolumn{4}{|c|}{ Heating } & \multicolumn{4}{|c|}{ Cooling } \\
\hline & $\begin{array}{l}\text { Temp. } \\
\text { contrac- } \\
\text { tion } \\
\text { began }\end{array}$ & $\begin{array}{l}\text { Temp. } \\
\text { contrac- } \\
\text { tion } \\
\text { ended }\end{array}$ & $\begin{array}{c}\text { Contrac- } \\
\text { tion }\end{array}$ & $\begin{array}{c}\text { Average } \\
\text { rate of } \\
\text { heating }\end{array}$ & $\begin{array}{l}\text { Temp. } \\
\text { expan- } \\
\text { sion } \\
\text { began }\end{array}$ & $\begin{array}{l}\text { Temp. } \\
\text { expan- } \\
\text { sion } \\
\text { ended }\end{array}$ & $\begin{array}{l}\text { Expan- } \\
\text { sion }\end{array}$ & $\begin{array}{l}\text { Average } \\
\text { rate of } \\
\text { cooling }\end{array}$ \\
\hline 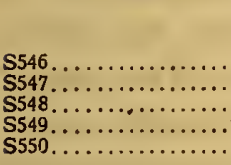 & $\begin{array}{l}-\mathrm{C} \\
742 \\
723 \\
722 \\
735 \\
729\end{array}$ & $\begin{array}{l}-\mathrm{C} \\
792 \\
771 \\
780 \\
790 \\
759\end{array}$ & $\begin{array}{r}\mu / \mathrm{m} \\
1255 \\
1495 \\
1440 \\
1495 \\
1605\end{array}$ & $\begin{array}{r}\bullet \mathrm{C} / \\
\text { minute } \\
0.38 \\
.80 \\
.37 \\
.71 \\
.73\end{array}$ & $\begin{array}{c}0 \mathrm{C} \\
682 \\
687 \\
6 \cdots .7 \\
746 \\
685\end{array}$ & $\begin{array}{l}{ }^{\circ} \mathbf{C} \\
661 \\
640 \\
665 \\
675 \\
659\end{array}$ & $\begin{array}{r}\mu / \mathrm{m} \\
1475 \\
1540 \\
130 . \\
1325 \\
1590\end{array}$ & $\begin{array}{r}{ }^{\circ} \mathrm{C} / \\
\text { minute } \\
0.49 \\
\ldots \ldots \ldots \\
\cdots \ldots \ldots .79 \\
.79\end{array}$ \\
\hline $\begin{array}{l}\mathbf{S} 551 \ldots \ldots \ldots \ldots \ldots \ldots \\
\mathbf{S} 552 \ldots \ldots \ldots \ldots \ldots \ldots \\
\mathbf{S} 5553 \ldots \ldots \ldots \ldots \ldots \ldots \\
\mathbf{S} 555 \ldots \ldots \ldots \ldots \ldots \ldots\end{array}$ & $\begin{array}{l}759 \\
744 \\
711 \\
730 \\
764\end{array}$ & $\begin{array}{l}806 \\
811 \\
790 \\
817 \\
826\end{array}$ & $\begin{array}{r}1910 \\
1535 \\
860 \\
740 \\
1135\end{array}$ & $\begin{array}{l}.57 \\
.67 \\
.84 \\
.76 \\
.54\end{array}$ & $\begin{array}{l}769 \\
763 \\
431 \\
781 \\
785\end{array}$ & $\begin{array}{l}717 \\
695 \\
332 \\
715 \\
704\end{array}$ & $\begin{array}{r}1805 \\
1605 \\
1515 \\
695 \\
1415\end{array}$ & $\begin{array}{l}.69 \\
.68 \\
.50 \\
.93 \\
.78\end{array}$ \\
\hline $\begin{array}{l}\mathbf{S} 556 \ldots \ldots \ldots \ldots \ldots \ldots \\
\mathbf{S} 557 \ldots \ldots \ldots \ldots \ldots \ldots \ldots \\
\mathbf{S} 558 \ldots \ldots \ldots \ldots \ldots \ldots \ldots \\
\mathbf{S} 559 \ldots \ldots \ldots \ldots \ldots \ldots \ldots\end{array}$ & $\begin{array}{l}734 \\
774 \\
806 \\
688 \\
738\end{array}$ & $\begin{array}{l}823 \\
858 \\
811 \\
742 \\
794\end{array}$ & $\begin{array}{r}1410 \\
700 \\
7730 \\
1310 \\
1310\end{array}$ & $\begin{array}{r}.82 \\
1.55 \\
.53 \\
.90 \\
.50\end{array}$ & $\begin{array}{r}806 \\
839 \\
\cdots \cdots \\
621 \\
746\end{array}$ & $\begin{array}{r}732 \\
757 \\
\cdots \cdots \\
542 \\
685\end{array}$ & $\begin{array}{r}90 \\
795 \\
7.20 . \\
1205 \\
1450\end{array}$ & $\begin{array}{r}.87 \\
2.35 \\
.82 \\
1.01 \\
.68\end{array}$ \\
\hline $\begin{array}{l}\mathbf{S} 561 \ldots \ldots \ldots \ldots \ldots \ldots \\
\mathbf{S} 562 \ldots \ldots \ldots \ldots \ldots \ldots \ldots \\
\mathbf{S} 563 \ldots \ldots \ldots \ldots \ldots \ldots \ldots \\
\mathbf{S} 564 \ldots \ldots \ldots \ldots \ldots \ldots \ldots\end{array}$ & $\begin{array}{l}758 \\
718 \\
694 \\
735 \\
864\end{array}$ & $\begin{array}{l}837 \\
787 \\
730 \\
770 \\
886\end{array}$ & $\begin{array}{r}820 \\
1300 \\
970 \\
1580 \\
605\end{array}$ & $\begin{array}{l}.65 \\
.50 \\
.61 \\
.72 \\
.92\end{array}$ & $\begin{array}{l}817 \\
730 \\
550 \\
738 \\
846\end{array}$ & $\begin{array}{l}738 \\
637 \\
540 \\
676 \\
805\end{array}$ & $\begin{array}{r}1010 \\
1200 \\
\cdots \cdots \\
13335 \\
835\end{array}$ & $\begin{array}{r}.67 \\
.83 \\
.56 \\
.90 \\
1.14\end{array}$ \\
\hline $\begin{array}{l}\mathbf{S} 566 \ldots \ldots \ldots \ldots \ldots \ldots \\
\mathbf{S} 507 \ldots \ldots \ldots \ldots \ldots \ldots \ldots \\
\mathbf{S} 482 \ldots \ldots \ldots \ldots \ldots \ldots \ldots \\
\mathbf{S} 504 \ldots \ldots \ldots \ldots \ldots \ldots \ldots\end{array}$ & $\begin{array}{l}725 \\
912 \\
732 \\
722 \\
790\end{array}$ & $\begin{array}{l}768 \\
917 \\
742 \\
833 \\
810\end{array}$ & $\begin{array}{r}1710 \\
1310 \\
1000 \\
1230 \\
\text { Indef. }\end{array}$ & $\begin{array}{l}.83 \\
.67 \\
.32 \\
.36 \\
.30\end{array}$ & $\begin{array}{l}721 \\
906 \\
712 \\
747 \\
775\end{array}$ & $\begin{array}{l}665 \\
903 \\
694 \\
648 \\
746\end{array}$ & $\begin{array}{r}1725 \\
163 \\
660 \\
1020 \\
2120\end{array}$ & $\begin{array}{r}.75 \\
.50 \\
2.78 \\
.89 \\
.54\end{array}$ \\
\hline $\begin{array}{l}\mathbf{S} 484 \ldots \ldots \ldots \ldots \ldots \ldots \\
\$ \$ \ldots \ldots \ldots \ldots \ldots\end{array}$ & $\begin{array}{l}\text { None } \\
\text { None }\end{array}$ & $\begin{array}{c}\text { None } \\
\text { (Coeff. } 8\end{array}$ & $\begin{array}{l}\text { None } \\
0 \text { to } 900=\end{array}$ & $17.7 ; 900$ to & $\ddot{1000}=18.9$ & 25 to 100 & $=1 \ddot{s}_{3} \ddot{3}$ & $\cdots \cdots$ \\
\hline
\end{tabular}

PART 4.-EXPANSION DATA

\begin{tabular}{|c|c|c|c|c|c|c|c|c|}
\hline \multirow{2}{*}{ Lab. No. } & \multicolumn{4}{|c|}{$\begin{array}{c}\text { Average coefficient of expansion }\left(\times 10^{6}\right) \\
\text { above critical region }\end{array}$} & \multirow{2}{*}{$\begin{array}{l}\text { Coefficient } \\
\text { on cooling } \\
600 \text { to } 25^{\circ} \mathrm{C}\end{array}$} & \multirow{2}{*}{$\begin{array}{l}\text { Increase } \\
\text { in length } \\
\text { at } 25^{\circ} \mathrm{C}\end{array}$} & \multirow{2}{*}{$\begin{array}{c}\text { Maxi- } \\
\text { mum dif- } \\
\text { terence in } \\
\text { beating } \\
\text { and } \\
\text { cooling } \\
\text { curves }\end{array}$} & \multirow{2}{*}{$\begin{array}{l}\text { Temp. } \\
\text { of maxi- } \\
\text { mum dif- } \\
\text { ference }\end{array}$} \\
\hline & Heating & $\begin{array}{l}\text { Temp. } \\
\text { rsnge }\end{array}$ & Cooling & $\begin{array}{l}\text { Temp. } \\
\text { range }\end{array}$ & & & & \\
\hline $\begin{array}{l}\mathbf{S} 546 \ldots \ldots \ldots \ldots \\
\mathbf{S} 547 \ldots \ldots \ldots \ldots \\
\mathbf{S} 548 \ldots \ldots \ldots \ldots \\
\mathbf{S} 549 \ldots \ldots \ldots \ldots \\
\mathbf{S} 550 \ldots \ldots \ldots \ldots\end{array}$ & $\begin{array}{r}23.4 \\
\ldots \ldots \ddot{2} . \overline{7} \\
22.8 \\
24.0\end{array}$ & $\begin{array}{c}\circ \mathrm{C} \\
825-900 \\
8900000 \\
800-900 \\
800-900\end{array}$ & $\begin{array}{l}22.7 \\
22.7 \\
22.7 \\
22.8 \\
23.1\end{array}$ & $\begin{array}{c}{ }^{\circ} \mathrm{C} \\
900-725 \\
900-700 \\
900-800 \\
900-800 \\
900-725\end{array}$ & $\begin{array}{l}14.5 \times 10^{-6} \\
14.6 \\
14.5 \\
14.4 \\
14.5\end{array}$ & $\begin{array}{l}-1202 \\
-462 \\
-\quad 110 \\
-\quad 82 \\
-\quad 400\end{array}$ & $\begin{array}{l}\mu / \mathrm{m} \\
-1250 \\
-630 \\
=125 \\
-145 \\
-465\end{array}$ & $\begin{array}{l}{ }^{\circ} \mathrm{C} \\
513-555 \\
485-515 \\
363-393 \\
505-525 \\
343-380\end{array}$ \\
\hline $\begin{array}{l}\mathbf{S} 551 \ldots \ldots \ldots \ldots \\
\mathbf{S} 552 \ldots \ldots \ldots \ldots \\
\mathbf{S} 553 \ldots \ldots \ldots \ldots \\
\mathbf{S} 554 \ldots \ldots \ldots \ldots \\
\mathbf{S} 555 \ldots \ldots \ldots \ldots\end{array}$ & 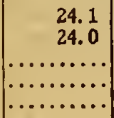 & $\begin{array}{r}825-900 \\
825-900 \\
\ldots \ldots \ldots \ldots \\
\ldots \ldots \ldots \ldots \\
\ldots \ldots \ldots \ldots\end{array}$ & $\begin{array}{l}22.8 \\
22.6 \\
22.3 \\
21.9 \\
22.0\end{array}$ & $\begin{array}{l}900-800 \\
900-800 \\
900-550 \\
900-800 \\
900-805\end{array}$ & $\begin{array}{l}14.3 \\
14.4 \\
10.7\left(275-25^{\circ} \mathrm{C}\right) \\
14.5 \\
14.2\end{array}$ & $\begin{array}{l}-270 \\
-\quad 90 \\
-680 \\
-198 \\
+343\end{array}$ & $\begin{array}{r}-355 \\
-195 \\
-225 \\
+380\end{array}$ & $\begin{array}{r}585-613 \\
640 \\
290 \\
405-423 \\
85-150\end{array}$ \\
\hline $\begin{array}{l}\mathbf{S} 556 \ldots \ldots \ldots \ldots \\
\mathbf{S} 557 \ldots \ldots \ldots \ldots \\
\mathbf{S} 558 \ldots \ldots \ldots \ldots \\
\mathbf{S} 559 \ldots \ldots \ldots \ldots \\
\mathbf{S} 560 \ldots \ldots \ldots \ldots\end{array}$ & $\begin{array}{r}\ldots \ldots \dot{2}, \\
\ldots \ldots \ldots\end{array}$ & 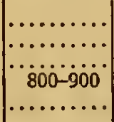 & $\begin{array}{r}\quad 23.0 \\
\cdots \cdots \cdots \cdots \\
\cdots \cdots \cdots \\
22.8 . \\
\cdots \cdots \cdots\end{array}$ & $\begin{array}{c}895-820 \\
\ldots \ldots \ldots \ldots \\
\cdots \ldots \ldots \\
900-700 \\
\ldots \ldots \ldots\end{array}$ & $\begin{array}{l}14.3 \\
13.9 \\
14.0 \\
13.1\left(500-25^{\circ} \mathrm{C}\right) \\
14.5\end{array}$ & $\begin{array}{l}-593 \\
+\quad 98 \\
+\quad 346 \\
+727 \\
+\quad 335\end{array}$ & $\begin{array}{l}-730 \\
+160 \\
+360 \\
+875 \\
+380\end{array}$ & $\begin{array}{r}675 \\
180-225 \\
90-160 \\
515-525 \\
125-195\end{array}$ \\
\hline $\begin{array}{l}\mathbf{S} 561 \ldots \ldots \ldots \ldots \\
\mathbf{S} 562 \ldots \ldots \ldots \ldots \\
\mathbf{S} 563 \ldots \ldots \ldots \ldots \\
\mathbf{S} 564 \ldots \ldots \ldots \ldots \\
\mathbf{S} 565 \ldots \ldots \ldots \ldots\end{array}$ & $\begin{array}{r}\cdots \cdots \\
\ldots \ldots \\
22.6 \\
z 3.0 \\
\cdots \cdots\end{array}$ & $\begin{array}{r}8 \ldots \ldots \ldots \\
800-900 \\
800-900 \\
\cdots \ldots \ldots\end{array}$ & $\begin{array}{r}22.7 \\
21.1 \\
23.0\end{array}$ & $\begin{array}{c}900-800 \\
900-600 \\
900-800 \\
\ldots \ldots \ldots \ldots\end{array}$ & $\begin{array}{l}14.1 \\
14.5 \\
10.9\left(500-25^{\circ} \mathrm{C}\right) \\
14.2 \\
12.1\end{array}$ & $\begin{array}{l}+\quad 18 \\
\Rightarrow \quad 200 \\
-920 \\
-\quad 148 \\
-\quad 63\end{array}$ & $\begin{array}{l}+110 \\
-225 \\
-2080 \\
-180 \\
-100\end{array}$ & $\begin{array}{l}182-223 \\
515-520 \\
525-530 \\
408-455 \\
570-615\end{array}$ \\
\hline $\begin{array}{l}\mathbf{S} 566 \ldots \ldots \ldots \ldots \\
\mathbf{S} 507 \ldots \ldots \ldots \\
\mathbf{S} 482 \ldots \ldots \ldots \\
\mathbf{S} 504 \ldots \ldots \ldots \ldots \\
\mathbf{S} 483 \ldots \ldots \ldots \ldots\end{array}$ & $\begin{array}{l}22.6 \\
23.4 \\
34 \\
23.4 \\
37\end{array}$ & $\begin{array}{l}800-900 \\
900-945 \\
750-875 \\
833-950 \\
815-900\end{array}$ & $\begin{array}{l}23.1 \\
23.4 \\
28.6 \\
21.6 \\
25\end{array}$ & $\begin{array}{l}900-800 \\
917-945 \\
875-713 \\
950-760 \\
900-775\end{array}$ & $\begin{array}{l}14.5 \\
13.3 \\
14.3 \\
14.8 \\
14.1\end{array}$ & $\begin{array}{r}-366 \\
-120 \\
\ldots .430 \\
+9040\end{array}$ & $\begin{array}{r}-366 \\
-1500 \\
-825 \\
-510 \\
+9340\end{array}$ & $\begin{array}{r}25 \\
912 \\
712 \\
600 \\
300\end{array}$ \\
\hline $\begin{array}{l}\mathrm{S} 484 \ldots \ldots \ldots \\
\mathrm{S} 457 \ldots \ldots \ldots\end{array}$ & & & & & $\begin{array}{l}13.6 \\
12.6\end{array}$ & $\because \ddot{0}$ & & \\
\hline
\end{tabular}


in Table 3. These results can be more readily interpreted by classifying them under the characteristics of the theoretical curve of Fig. I. This curve represents the critical regions within which

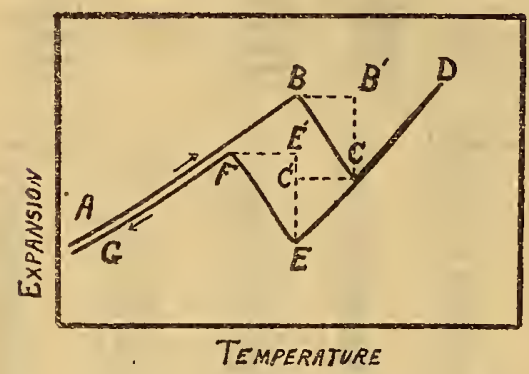

FIG. I. - Theoretical expansion curve for range 600 to $900^{\circ} \mathrm{C}$ anomalous length changes occur. Most of the specimens followed the sequence of expansion as shown by the curve $A, B, C, D, E$, $F, G$ of this figure. The length increments were usually quite regular up to point $B$, where they reversed sign and were recorded as contractions until point $C$ was reached. From point $C$ the expansion was usually greater than at any previous point. On cooling from $D$ to $E$ the contraction corresponded to the expansion on heating. At $E$ the contraction ceased and an expansion was recorded until point $F$ was approached. Below this point the contraction was again similar to the corresponding expansion recorded on heating.

The values for vacuum electrolytic iron ${ }^{3}\left(\mathrm{~S}_{50} \mathrm{O}\right.$, Fig. 2) may serve as reference values for the alloys. The temperature interval of contraction with heating $\left(B, B^{\prime}\right.$ of Fig. $\left.\mathrm{r}\right)$ extended from 912 to $917^{\circ} \mathrm{C}$. The corresponding interval of expansion with cooling $\left(E^{\prime}, F\right)$ extended from 906 to $903^{\circ} \mathrm{C}$. These intervals of temperature represent the retardations due to in-

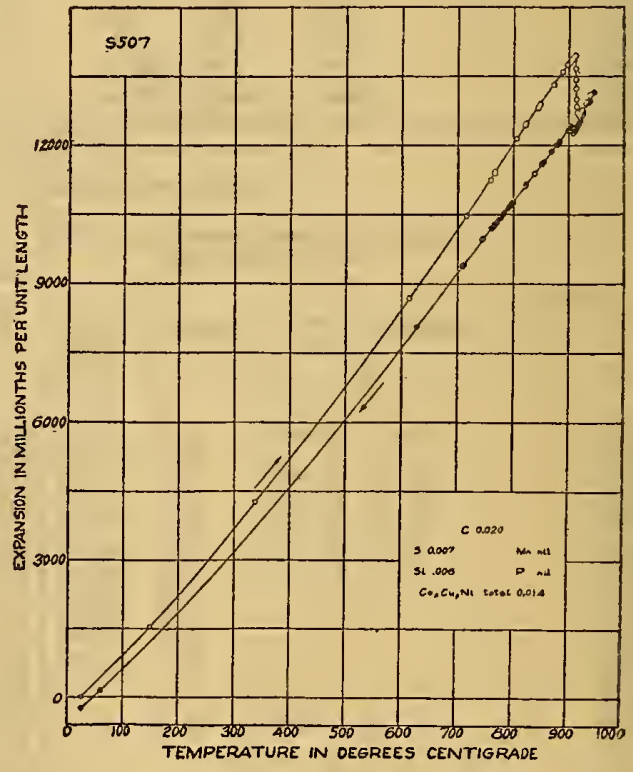

FIG. 2.-Expansion curve for electrolytic iron herent resistances to this change, to impurities present, to our finite rate of heating or cooling. Where the retardations exceed these limits they may be assumed to be the results of alloying elements. 
The temperature interval between points $C$ and $E\left(C C^{\prime}\right)$ is not constant and varies fron I I $^{\circ} \mathrm{C}$ for iron to an indefinite value of about $350^{\circ} \mathrm{C}$ on specimen $\mathrm{S}_{553}$ (Fig. 3). Specimen $\mathrm{S}_{4} 84$ (Fig. 4) is not included in this comparison because of the lack of proper temperature range necessary to bring out these differences.

The low expansivity of invar at room temperatures is due to the fact that this $E, F$ region has been lowered to corresponding temperatures. Other specimens of nickel steel showing this lowering of the $E, F$ region are: $\mathrm{S}_{559}$ (Fig. 5) and $\mathrm{S}_{5} 63$ (Fig. 6). These curves, including $\mathrm{S}_{4} 84$, give their relations to other steels at high temperatures. Excluding nickel steels, the greatest displacements are given by $\mathrm{S}_{546}$ (Fig. 7), $\mathrm{S}_{547}$ (Fig. 8), and $\mathrm{S}_{504}$ (Fig. 9). On $555^{6}$ (Fig. Io) this difference is only $17^{\circ} \mathrm{C}$, whereas on $\mathrm{S}_{546}$ (Fig. 7 ) it amounts to $\mathrm{I}^{\circ} \mathrm{O}^{\circ} \mathrm{C}$. Now, the usual procedure in hardening steel is to quench from some temperature a certain number of degrees above the temperature of the irregularities of the heating curve. Should it develop that the most desirable quality is present in the steel at point $E$ (after having passed point $C$ ) rather than at point $C$, it is conceivable that errors approaching $100^{\circ} \mathrm{C}$ may have entered the quenching values.

Alloys showing the maximum differences between temperatures $B$ and $C\left(B B^{\prime}\right)$ are $\mathrm{S}_{50} \mathrm{O}_{4}$ (Fig. 9), $\mathrm{S}_{55} 6$ (Fig. IO), and $\mathrm{S}_{554}$ (Fig. I I). Similarly between $E$ and $F\left(E^{\prime} F\right)$ we have $\mathrm{S}_{504}$ (Fig. 9), $\mathrm{S}_{562}$ (Fig. 12), and $\mathrm{S}_{553}$ (Fig. 3). Cast iron is excluded because of the indefiniteness due to growth.

Perhaps one of the most important variations is that of length change between $E$ and $F\left(E E^{\prime}\right)$. If we assume the usual stressstrain relations as applying in this case then the stresses set up within a specimen, the outer shell of which has passed through this range before the inner, will be proportional to the length $\left(E E^{\prime}\right)$. This feature will be discussed later in connection with the results of a hardened specimen (S614). Specimens S55 I (Fig. 13) and $S_{5} 66$ (Fig. 14) illustrate this maximum expansion on cooling. The rate at which this stress is applied depends upon the value of $E E^{\prime}$, the value of $E^{\prime} F$, and the rate of cooling of the different sections of the specimen.

It is interesting to note that the specimen of high silicon steel $\mathrm{S}_{457}$ (Fig. 15) gave only slight if any reversals although heated to $1002^{\circ} \mathrm{C}$.

The coefficient of expansion of iron over the range 25 to $100^{\circ} \mathrm{C}$ is $12.0 \times 10^{-6}$. The average value for 25 of the steels tested is I $1.2 \times 10^{-6}$, and the deviations (excluding high chromium and 


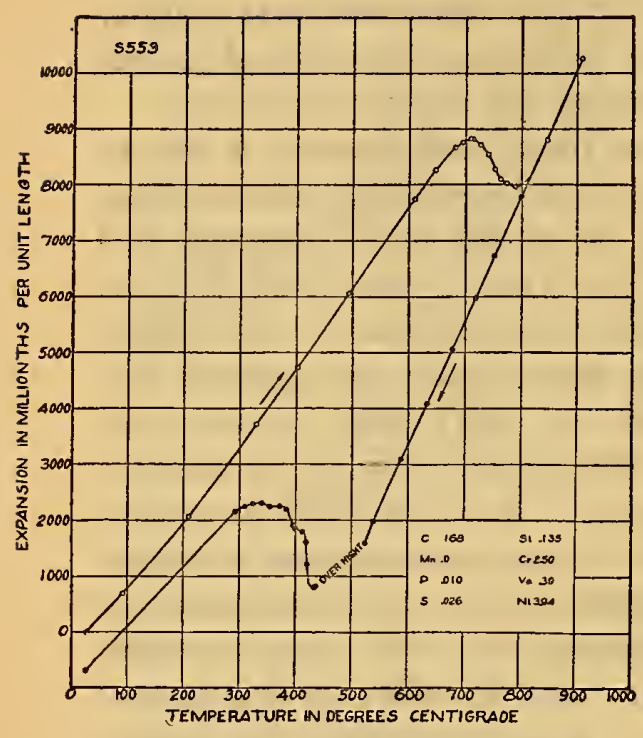

FIG. 3.-Expansion curve for nickel steel.

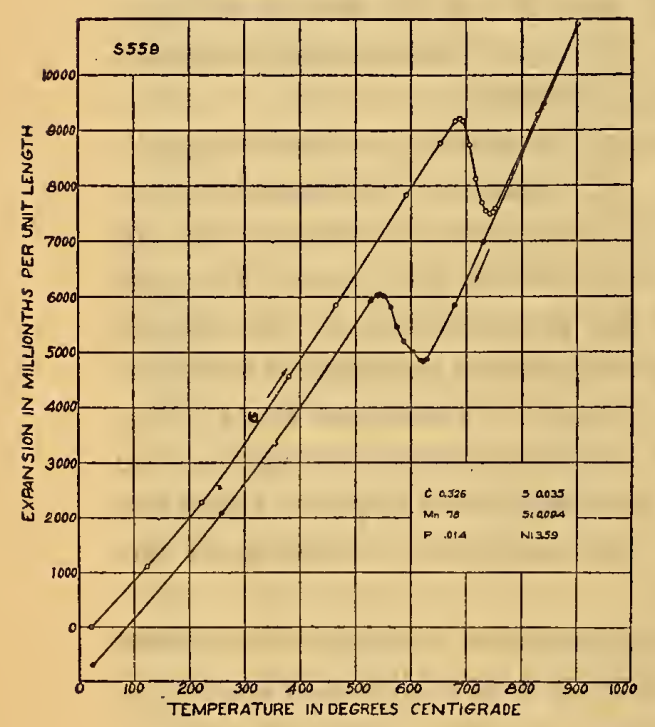

FIG 5.-Expansion curve for nickel steel

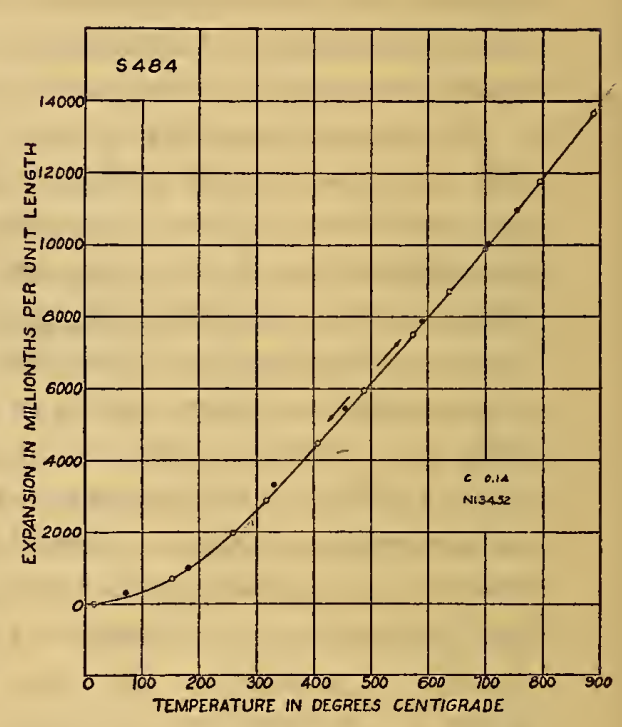

Firg. 4.-Expansion curve for nickel steel

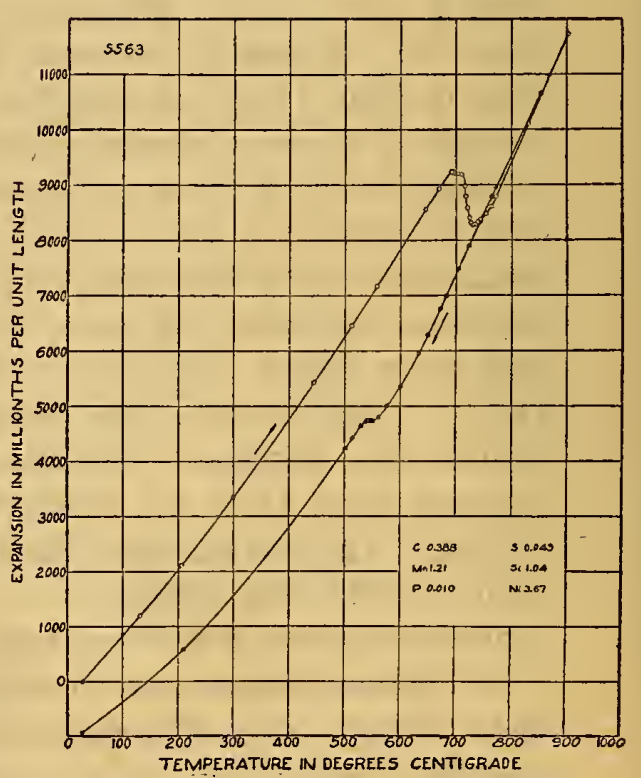

Fic. 6. - Expansion curve for nickel-silicon steel 


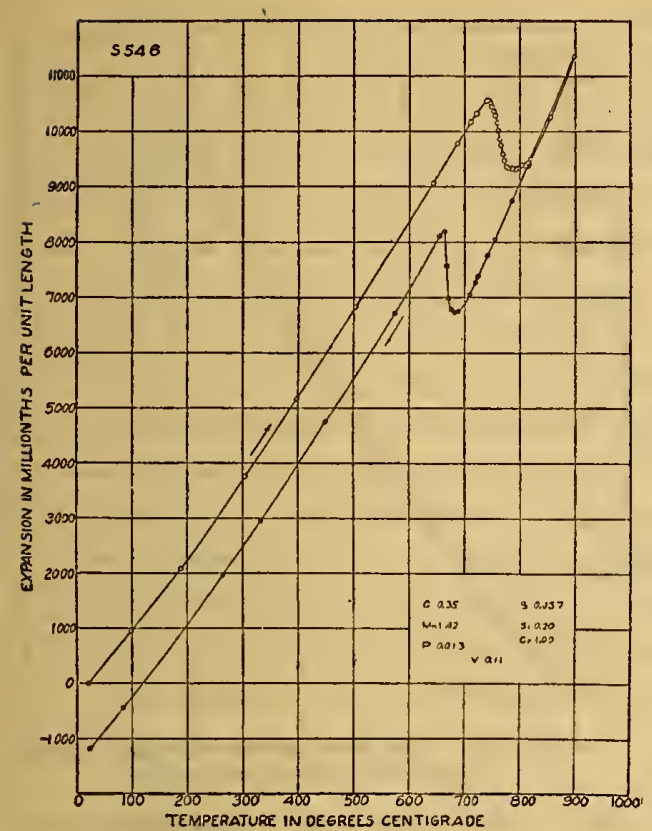

FIG. 7.-Expansion curve for steel showing lorge zariations between heating and cooling critical regions

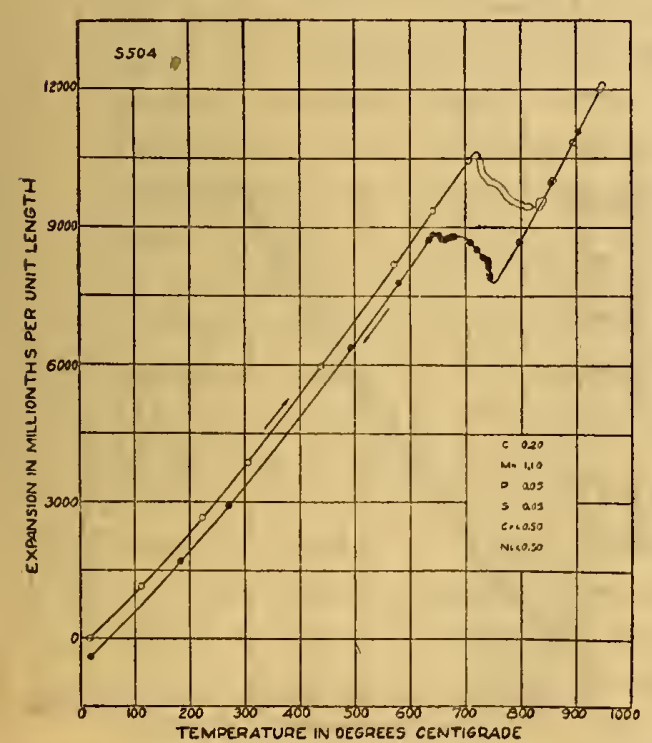

FrG. 9. Expansion curve for stecl showing wide critical regions

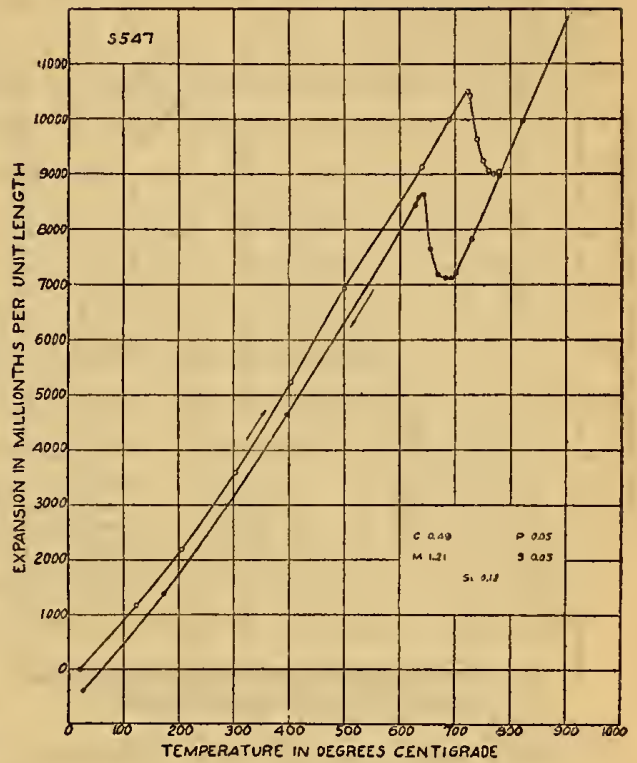

FIg. 8.-Expansion curve for steel showeing large variations between heating and cooling critical regions

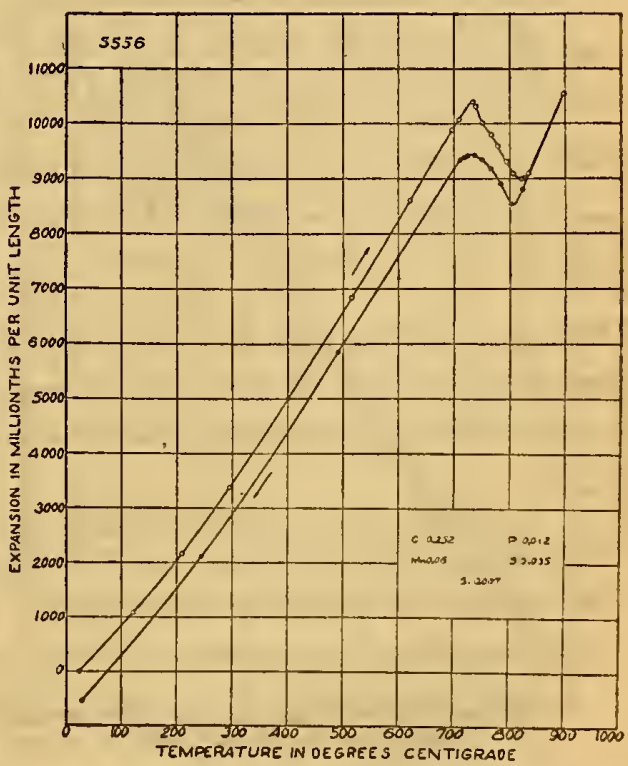

Fic. 10.-Expansion curvc for steel shouing wide critical regions 


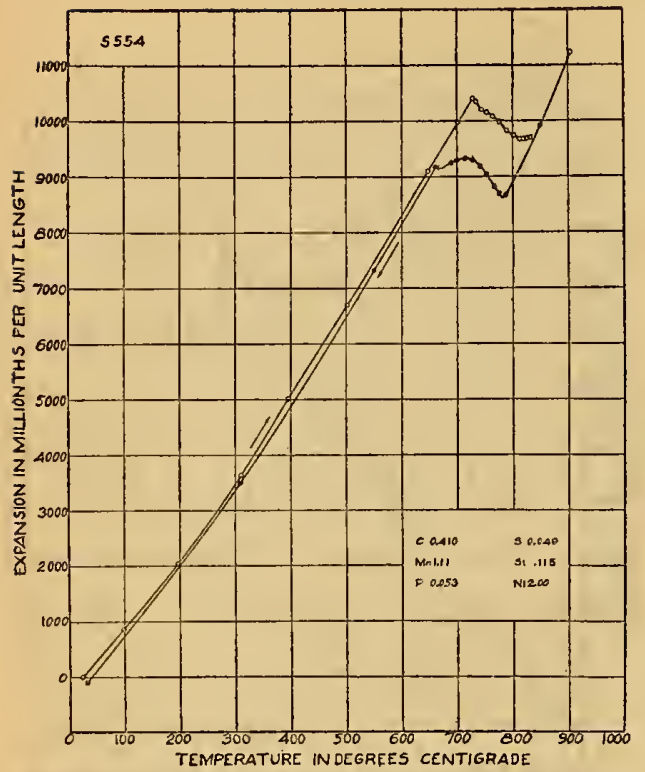

FIG. II.-Expansion curve for steel showing wide critical regions

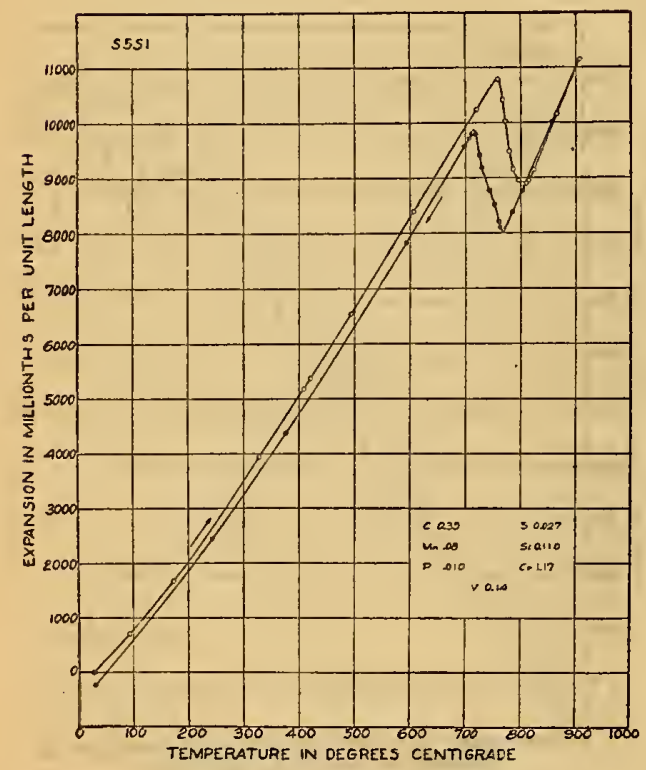

FIG. I3.-Expansion curve for steel showing large change of dimension at critical regions

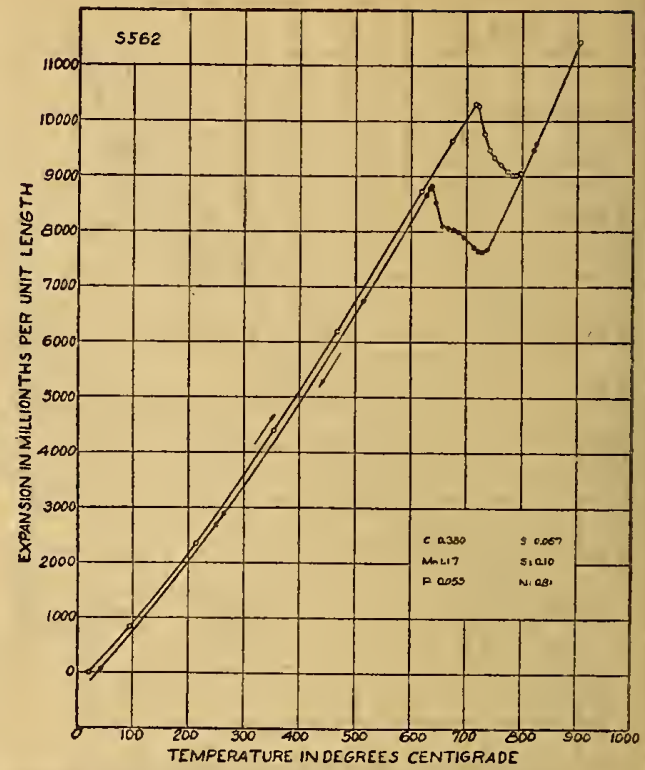

FIG. I2.-Expansion curve for steel showing wide critical regions

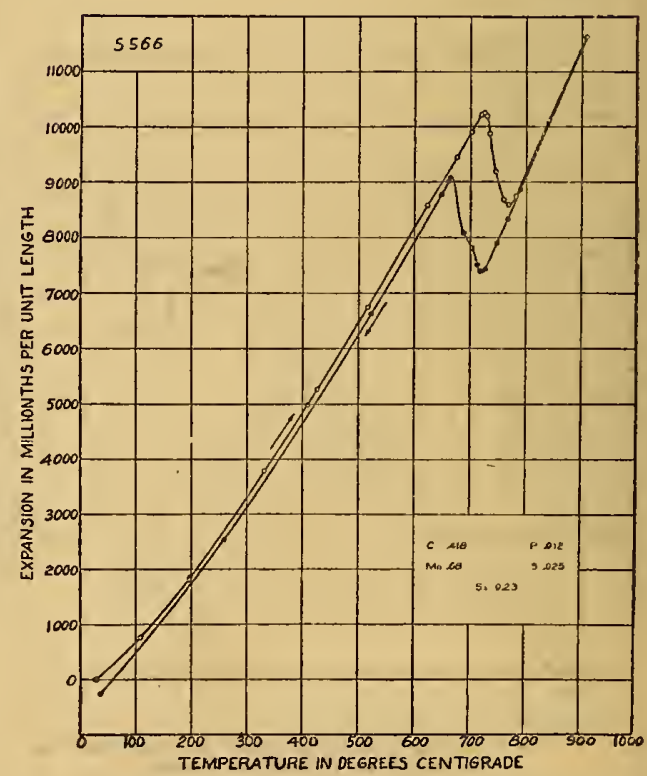

FIG. I4.-Expansion curve for steel showing large change of dimension at critical regions 
nickel steels) are small. The average value from 25 to $600^{\circ} \mathrm{C}$ is $14.2 \times 10^{-6}$. Above the critical regions the values jump to values between $22.6 \times \mathrm{xO}^{-6}$ and $24 . \mathrm{I} \times \mathrm{xO}^{-6}$. The special high carbon, nickel, and silicon steels are not included within this range. The cooling coefficients are closely related. Within the critical region the coefficient is quite different, usually reversing sign. This reversal of sign, or expansion on cooling, may set up stresses in the hardened steel which if not properly released will cause cracking or distortion. The higher the temperature above the critical region from which the specimen is quenched and the faster the rate of cooling, the greater should be the tendency to these defects. Undoubtedly the coarser grain structure of the higher temperatures has some influence; but the cooling of the outer layer before the interior cools will set up stresses, since this outer layer adapts itself about the interior before the interior has passed through the critical region where it will tend to expand, and to stretch or crack the outer shell. The release of this stress of outer shell is shown in S6r4 (Fig. r6). The relative amount of this stress can be compared in the column headed "Expansion $\mu / \mathrm{m}$ (cooling)."

The works of Honda, ${ }^{10}$, Chevenard, ${ }^{11}$ and others show that quenching steel may carry point $E$ several hundred degrees below the normal slow-cooling position recorded in our tests. If we apply the coefficient $23 \times 10^{-6}$ for the contraction rate over these several hundred degrees range for the retarded or undercooled phase of the outer surface, and apply the normal coefficient (approximately) $16 \times 10^{-6}$ for the inner portions where the cooling has been sufficiently slow to permit the transformations, we justify an intensification of the above stresses.

When variations in the ratios of quenching of different parts of a surface exist, the above-mentioned inequalities in the time and rate of expansion or contraction may readily manifest their effects in warping or local cracking.

Steel No. S6r4, Fig. 16, is included to show the effects of hardening as related to length changes. This specimen was heated to $850^{\circ} \mathrm{C}$ and quenched in oil. Upon heating above $50^{\circ} \mathrm{C}$ we found the curve departing from the annealed-steel curve (see dotted lines for average annealed steel). The specimen was carried from 50 to $100^{\circ} \mathrm{C}$ and back in about 200 minutes and gave a shrinkage of about 250 microns per meter. This shrink-

\footnotetext{
${ }^{30}$ Honda, Tôkohu University, Scientific Reports, 8, pp. I8r-205. Honda, Matsushita and Iơei, J. Iron and Steel Inst., 108 , pp. $25 x-269$.

31 P. Chevenard, C. R., 165, pp. 59-6z; Rev. de Mét., 16, pp. r7-rg.
} 


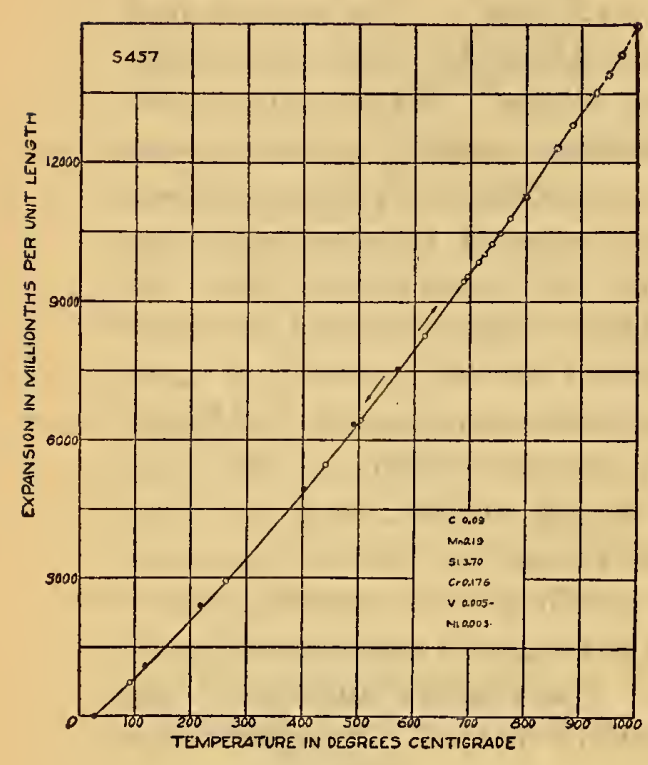

FrG. 15. - Expansion curve for silicon steel

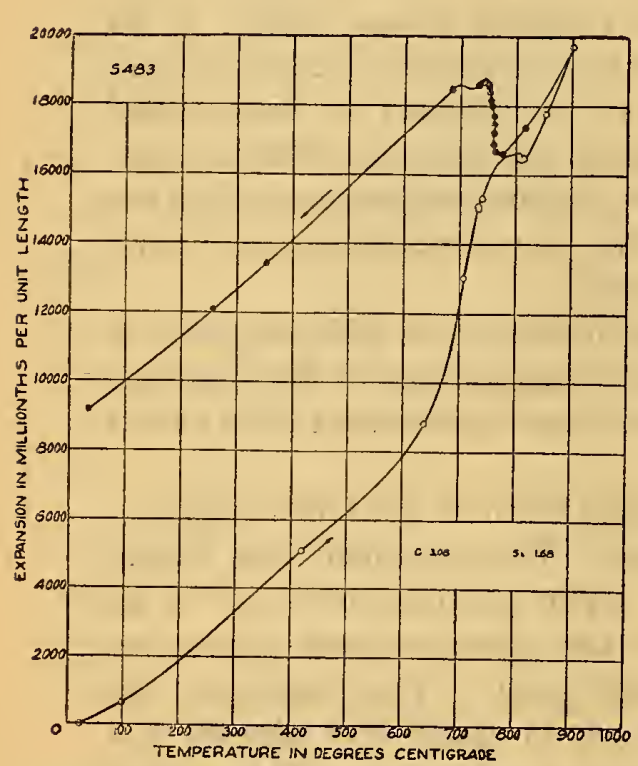

IIG. I7.-Expansion and growth curve for cast iron

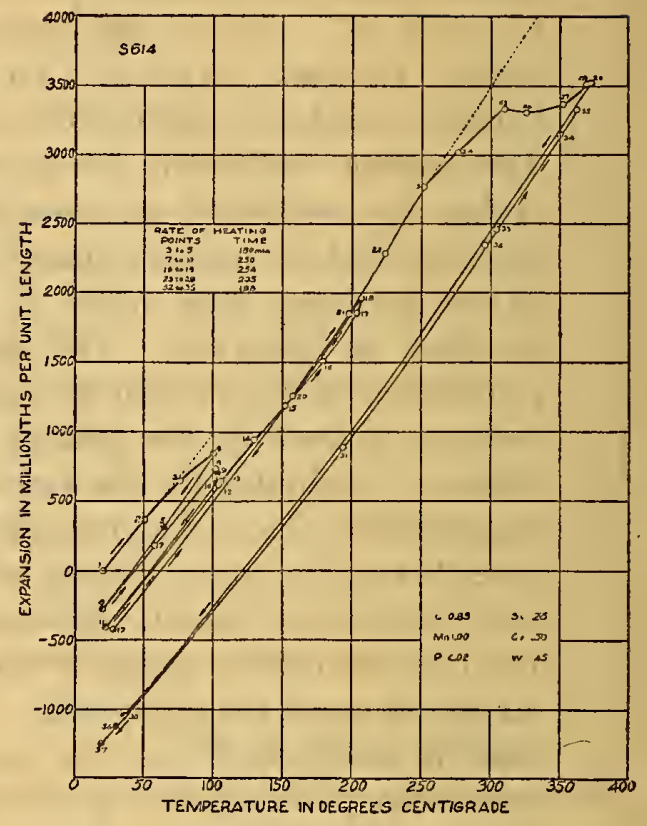

Iirg. I6.-- Expansion curve for steel showing relcase of strains in draving

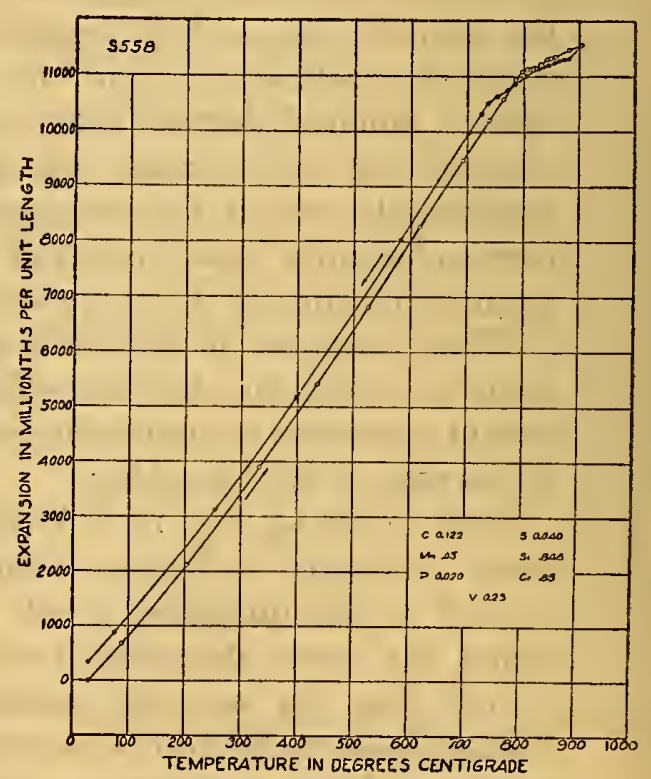

FIg. I8.- Expansion curve for stecl, showing small change of dimension at critical regions 
age is to be expected since the outer shell was stressed by the central portions passing through the (expanding) critical regions after the outer layers had passed this stage and had started on the regular contraction. Reheating to $100^{\circ} \mathrm{C}$ and holding for about 4 hours was sufficient to release additional stresses, and the specimen then showed a total shrinkage at $25^{\circ} \mathrm{C}$ of 400 microns per meter, or in more general terms 400 millionths per unit length. This heat treatment appears to have released practically all the stresses possible to release at or near $100^{\circ} \mathrm{C}$, for additional heating to $200^{\circ} \mathrm{C}$ gave very little additional shrinkage. The cooling curve from $200^{\circ} \mathrm{C}$ crosses the heating at about $150^{\circ} \mathrm{C}$; no explanation is evident for this phenomenon. Reheating to $250^{\circ} \mathrm{C}$ and above renewed the shrinkages, and at 310 to $350^{\circ} \mathrm{C}$ the effect was very pronounced. At $370^{\circ} \mathrm{C}$ the effect appeared to be complete and upon return to room temperature gave a total shrinkage of I250 microns per meter, or one-eighth of i per cent.

This shrinkage has been shown for a specimen of chrome steel ${ }^{12}$ on which the observations were carried beyond the critical ranges. The present curve gives greater detail of the changes.

The increase or decrease in length on cooling to $25^{\circ} \mathrm{C}$ was usually small and, in a large degree, indicates the lack of perfect annealing before the test was started. The maximum differences between the heating and cooling curves are closely related to the permanent length changes.

Figs. I $8,19,20,2 \mathrm{I}$, and 22 are included as additional specimens which emphasize the differences previously mentioned.

\section{SUMMARY}

I. Data on the anomalous expansion of a few steels and irons have been recorded, and made available for reference.

2. The expansion of iron has been determined over the range 25 to $945^{\circ} \mathrm{C}$. The expansion from 25 to $100^{\circ} \mathrm{C}$ was found to be $12.0 \times 10^{-6}$. The average expansion of a number of steels, over this same range was found to be $1 \mathrm{I} .2 \times 1 \mathrm{IO}^{-6}$, and for the range 25 to $600^{\circ} \mathrm{C}, \mathrm{I} 4.2 \times 10^{-6}$.

3. The coefficient for the ordinary steels which we have heated above the critical regions have been evaluated as approximately $23 \times 10^{-6}$.

4. The shrinkages and warpages of drawn steels have been interpreted in terms of the rate of expansion and rate of temperature reduction on passing through the critical regions.

WASHINGTON, November 8, I92 I.

12 B. S. Sci. Papers, No. 426, p. 513. 